\title{
Electromagneto-Optical Scanning of the Magnetic Domains Local Areas of Bismuth-Substituted Yttrium Iron Garnets
}

\author{
Vadim E. Koronovskyy, Alehander A. Kalenichenko \\ Department of Radiophysics, Taras Shevchenko Kiev National University, Kiev, Ukraine \\ E-mail:koron@univ.kiev.ua \\ Received March 11, 2011; revised May 11, 2011; accepted May 20, 2011
}

\begin{abstract}
Magneto-electric properties of the magnetic domains local areas of bismuth-substituted yttrium iron garnet films are investigated. The electromagneto-optical (EMO) scanning method was used in our experiments when probing by laser beam various sites of separate magnetic domains of the film. Registered in our experiments the nonlinear and linear components of EMO effect does not remain to constants at optical scanning of various points of the magnetic domain, i.e., the local EMOE picture from the separate sites of the domain must be more informative than an averaged one in the multidomain case.
\end{abstract}

Keywords: Magneto-Electric, Electromagneto-Optical, Domain, Yttrium Iron Garnet

\section{Introduction}

Experimental and theoretical research on iron garnets as a large class of ferrimagnetic oxides with high Curie temperature $\left(T_{C}>500 \mathrm{~K}\right)$, has a long history $[1,2]$. Some distinct advantages of yttrium iron garnet $Y_{3} F_{5} O_{12}(\mathrm{YIG})$ are that this material can be grown in very pure crystals and has a narrow ferromagnetic resonance line, indicating very low spin-wave damping. Actually, YIG has a rather complicated crystal structure with space group $I a 3 d$ and 20 magnetic ions in the primitive cell. Single crystal YIG films grown by liquid phase epitaxy were brought to a high level of development as materials for bubble memory devices and remain interesting for microwave applications until now. Nonmagnetic gadolinium gallium garnet has nearly the same lattice constant as YIG, and has for this reason become the prime substrate for epitaxial growth of garnet films. It has been known for a long time that the magnetic garnets have a region of relative transparency in the infrared wavelength region between 1.5 and $5 \mu \mathrm{m}$ [3]. For this reason, they are considered as materials for integrated optics, where garnet lasers, isolators, and modulators are arranged on the same substrate [4]. Due to the strong magneto-optical Faraday and Kerr effects, YIG and rare earth iron garnets can be also instrumental as magneto-optical indicator films as well as magnetic field sensors. Substitution of part of the $Y^{3+}$ on dodecahedral sites by the diamagnetic $B i^{3+}$ has been found to increase the Curie temperature. It also has the effect of strongly enhancing the magnetooptical properties of YIG, in particular, the linear magneto-optical response. For this reason bismuth-substituted yttrium iron garnets have had an important impact in the area of magneto-optic materials. Bismuth substitution is known to increase the refractive index of garnets in the low energy region of the spectrum and to increase the absorption $[5,6]$.

The sensitivity of the magneto-optical response of garnet (bismuth-substituted yttrium iron garnet) films to an external field is one of the main properties of interest for imaging applications. It is commonly investigated by measuring the field dependence of the Faraday rotation in the spectral region of interest. This, however, reflects only averaged bulk properties of the investigated film. Critical to understanding the magnetic properties and the technological applications of YIG or bismuth-substituted YIG is the ability to observe and measure magnetic domain structure and separate magnetic domain. The magneto-optic method allows us to read out a domain pattern and at the same time to follow the kinetics of formation, motion, and annihilation of these domains.

After experimentally founding by O'Dell of the magneto-electric effect (MEE) in YIG [7] (MEE - the effect of change of a magnetic state in a solid under the action 
of an external electric field) the active researches of magneto-electric properties of this ferrimagnetic have begun. In our earlier investigations, MEE in YIG films was studied with using the optical polarimetry method [8-10].

In the current paper we inform about results of the further researches of the MEE by polarimetry method in separate magnetic domains of the bismuth-substituted YIG films. This method deals with the registration of the changes in magneto-optical Faraday rotation of the light polarization plane under the action of electric field applied to the film. So, the effect of light polarization plane rotation in crystals induced by the external electric field was determined as the electromagneto-optical (EMO) effect (EMOE) [11]. EMOE is one of the approaches to the investigation of magneto-electric phenomena in garnet films.

\section{Experimental Procedure}

Our experimental technics as combination of highsensitive laser polarimetry and a polarising microscope in details described in Refs. 8. This technics allows us to measure a local values of the changes of light polarization plane rotation (Faraday rotation) under action of variable electric field applied to the sample ( $\alpha_{\text {Емо }}$ parameter from separate sites of the magnetic domain) and the integral EMO effect, from multidomain sites of the film. In our experiments as the starting point, we use bismuth-substituted yttrium iron garnet film grown by liquid phase epitaxy on $0.6 \mathrm{~mm}$ thick gadolinium gallium garnet substrate. Thickness of the film was $9 \mu \mathrm{m}$ range. A variable voltage, with a frequency $\omega=1000 \mathrm{~Hz}$ and a static magnetic field could be applied to the sample. Experiments were carried out at room temperature in longitudinal geometry $(\mathbf{E}\|\mathbf{k}, \mathbf{H}\| \mathbf{k}$, where $\mathbf{k}$ is a light wave vector, $\mathbf{H}$ is a static magnetic field, $\mathbf{E}$ is an electric field). The domain magnetisation of the film was normal to the film plane. A He-Ne laser $(\lambda=0.63 \mu \mathrm{m})$ was used in the experiments and using of a round diaphragm [8] allowed us to allocate the sites of a film of about $3 \mu \mathrm{m}$ in diameter.

Our experimental setup allowed us to carry out simultaneously measurements of linear $\left(\alpha^{\omega}\right)$ and nonlinear $\left(\alpha^{2 \omega}\right)$ components of the EMOE. The linear component registered on the basic frequency of an electric field and nonlinear component registered on the double frequency of the E-field (square law on electric field component).

We already informed, that at local measurements of the EMOE in magnetic domains of YIG films, the effect was registered both in domains with a magnetisation direction coinciding with a magnetic field direction ( $\mathrm{H} \uparrow \uparrow \mathrm{M}$, where the domain volume grows in magnetic field), and in domains with a direction of magnetisation against a magnetic field ( $\mathrm{H} \uparrow \downarrow \mathrm{M}$, where the domain volume decreases in magnetic field) [8]. At measurements was not observed dependences of the EMOE from magnetic field in wide range of its change. Dependence of the EMOE from magnetic field in domains of both signs started to be essential, in a vicinity of a field of magnetic saturation. In the current paper we do not consider the specified area of magnetic field.

\section{Results and Discussion}

In Figure $\mathbf{1}$ are presented magnetic-field dependencies of EMOE, measured in separate domains of investigated film, which increase (1) and decrease (2) the volumes with growth of the magnetic field. As we can see from presented curves, in a wide range of change of a magnetic field (before the transition of the film to the homogeneous magnetization), the effect practically does not change at measurements in both domains. The EMOE is connected with the influence of the external electric field on the magnetic anisotropy parameter of the film [8]. Electric field induce additional anisotropy which is small in comparison with magneto-crystal anisotropy [12]. The detected EMO signals are correlated to the modifications of domain structure induced by the electric field because of high sensitivity of domain structure to the changes in the magnetic anisotropy of the film. The registered effect is more value in comparison with similar local measurements in YIG [8]. Presence in our experimental setup of a polarising microscope and a scanning diaphragm which can allocate on the sample small sites in diameter about 3 $\mu \mathrm{m}$, allows us to spend measurements of EMOE on various sites of the separate domain. The experimental setup also allowed us to carry out simultaneously measurements of the square law on the E-field component of EMOE $\left(\alpha^{2 \omega}\right)$ and the linear component $\left(\alpha^{\omega}\right)$.

In Figure 2 one of the received dependences of EMOE from positions of scanning diaphragm (X) in the central areas of separate magnetic domain is presented. When we spent measurements a linear $\left(\alpha^{\omega}\right)$ and not linear $\left(\alpha^{2 \omega}\right)$ components of the electric-field-induced changes of the Faraday rotation were supervised simultaneously. Measurements were spent at $\mathrm{H}=0 \mathrm{Oe}$ and $\mathrm{E}=3,2 \mathrm{kV} / \mathrm{cm}$ ( 1 and 3 curves) and at $\mathrm{H}=140 \mathrm{Oe}$, $\mathrm{E}=3,2 \mathrm{kV} / \mathrm{cm}$ (curve 2) at moving of a scanning diaphragm in longitudinal direction in relation to positions of domain walls (DWs). We can see from the presented curves that the received results of measurements have some important features.

1) At $H=0$, the EMOE is nonzero at a single domain sites.

2) The nonlinear (square law on the electric field) component of EMO signal in some points of optical scanning differs from a signal average level on the domain. 


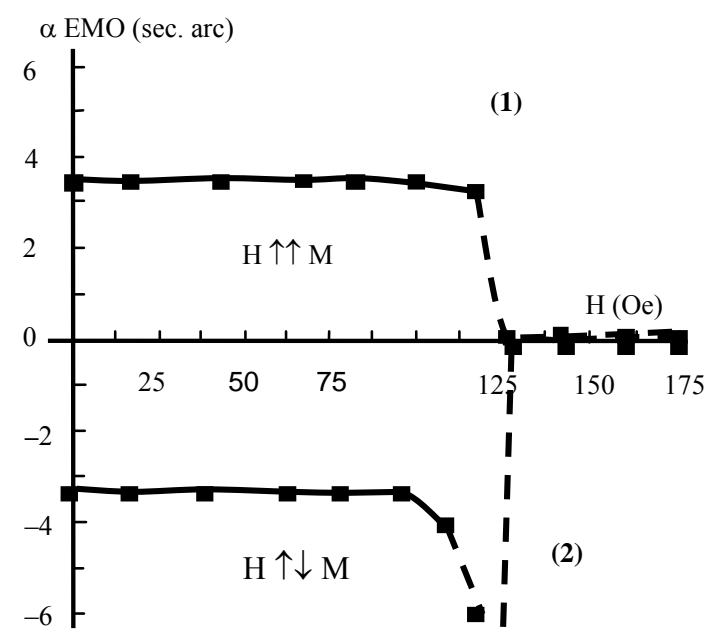

Figure 1. Magnetic-field dependencies of EMOE measured on the domains, which increase (1) and decrease (2) the volumes in magnetic field.

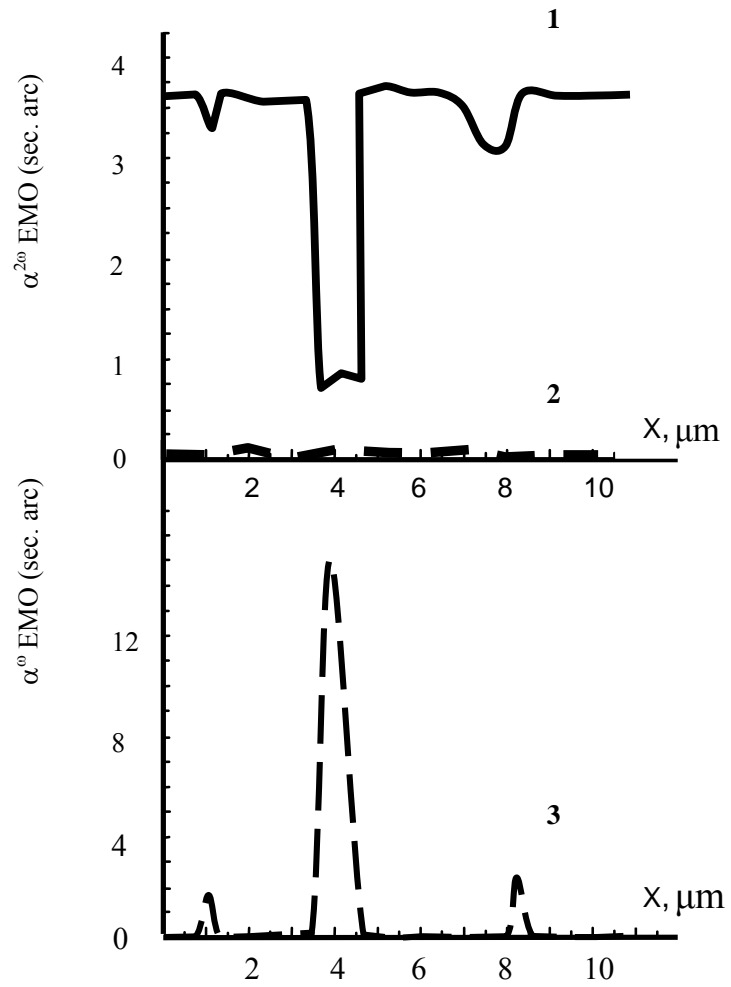

Figure 2. Dependences of the nonlinear (1) and linear (3) components of EMOE from positions of scanning diaphragm $(\mathrm{X})$ in the central areas of separate magnetic domain when $H=0$ Oe and $E=3,2 \mathrm{kV} / \mathrm{cm}$. Curve (2) was measured when $\mathrm{H}=140 \mathrm{Oe}, \mathrm{E}=3,2 \mathrm{kV} / \mathrm{cm}$.

3) In several points of a film, within the investigated domain, the linear component of EMOE was registered by us in the form of separate narrow peaks.

4) For the large fields, when the film magnetization saturates, the EMO signal decreases to values close to zero (Figure 2, curve 2).

EMO effect measurements in others, any way chosen domains, have shown similar results on character of EMOE dependence. Differences were observed only in density, localisation and width of a signal change peaks.

The received experimental results, in our opinion, have a following explanation. On the separate domain, as on the area with homogeneous magnetisation, the EMO effect of invariable value can be registered [8]. But, as we can see from Figure 2, stability of EMOE in different areas of the domain is broken. We have registered drastic decrease of square law on the electric field component of EMO signal in some separate points of the film within the investigated magnetic domain. As it has already been shown by us earlier, $\alpha_{\text {Емо }}$ parametre, at invariable magnetisation depends on the value of demagnetization fields in vicinities of a point of laser probing. It has also been shown that magnetization, which mainly defines the light polarization plane rotations, does not change under the electric-field action in YIG [8]. Such drastic decrease of square law on the electric field component of EMO signal are probably can be connected to the changes of the local value of demagnetizing fields in the small scanning areas, as just this component of Faraday rotation (at invariable magnetisation in electric field) is modulated by the variable electric field in our experiments. It can be in the film areas or in separate film points with not uniform magnetisations. In such areas we have the changeable local values of a demagnetization fields. Defects on a surface or in film volume can be such areas, most likely, as EMO signal changes occurs in the form of separate narrow peaks. Defect breaks a continuous condition of material and the local magnetisation difference is formed on it. Magnetisation directions in vicinity of defects can essentially differ from crystallo-graphic directions of easy magnetisation for considered material. i.e., the combination of local demagnetization fields and not uniformity of parametres can lead to registration of the EMOE local changes in separate areas in the domain (Figure 2).

Occurrence of linear on the electric field component of EMO signal in some points of a film (Figure 2), in our opinion, has a following explanation. In our earlier studies, only square-law on the electric field EMOE was observed in separate areas of YIG and bismuth-substituted YIG films (without additional external influence on the sample) [8-10]. The MEE and EMOE that are linearly dependent on the electric field are forbidden for the centrosymmetric cubic crystal structure of garnets and only a square-law on the electric field effect is possible [13]. But EMO signal occurrence on the basic frequency of 
electric field (linear on the electric field component of EMOE), allows us to assume that at least some of the investigate film's sites have non-centrosymmetric structure. Apparently from a Figure 2, in these points sharply decreases square law on the electric field component of EMO signal and under our assumption it is local areas or separate points of defects.

In Reference [14], we reported the results of some experimental investigations of the thermal influence on magneto-electric characteristics of epitaxial YIG films. Considering high sensitivity of the EMOE to changes in structural characteristics of epitaxial films, we have executed out investigation of thermal influence on EMOE in separate domains of bismuth-substituted yttrium iron garnets. Thermal influence on the sample was spent at temperature $\mathrm{T}=753 \mathrm{~K}$ throughout 240 minutes with the subsequent slow cooling. The experiment similar to previous (see Figure 2) has been made by us, at the same values of magnetic and electric field. In Figure 3 one of the received dependences of EMOE from positions (X) of scanning diaphragm in the central areas of any way chosen separate magnetic domain are presented. It is possible to see from curves (Figure 3) that general character of EMO displays in the domain has not changed essentially after thermal influence. Instability of a square law on the electric field component of EMO signal is observed. The linear component of EMOE was registered by us too in several points of a film. Thus, unlike measurements of the integral EMOE on multidomain sites of a garnet film [14], the received results of local measurements in separate domains do not allow us to track effect of temperature influence on magneto-electric characteristics of bismuth-substituted yttrium iron garnet films. We assume that the low-temperature mode chosen by us not sufficient for activation of processes of destruction of chemical bonds and deformations of a crystal lattice at thermally stimulated processes of migration of ions in a crystal lattice that could be shown through change of local EMO effects. Possible relaxation of interfacial strain for the film surface and interface regions after temperature influence can be registered at measurements of the EMOE from multidomain areas.

\section{Conclusions}

Thus, in our experiments when probing by laser beam various sites of separate magnetic domain of the bismuth-substituted yttrium iron garnet film and using EMO method, we registered the nonlinear (square law on the electric field) and linear components of EMOE. Local areas or separate points on a surface or in film volume where sharp changes of EMO signal were registered, under our assumption can be areas of defects of the sample. When scanning various sites of the magnetic domain,

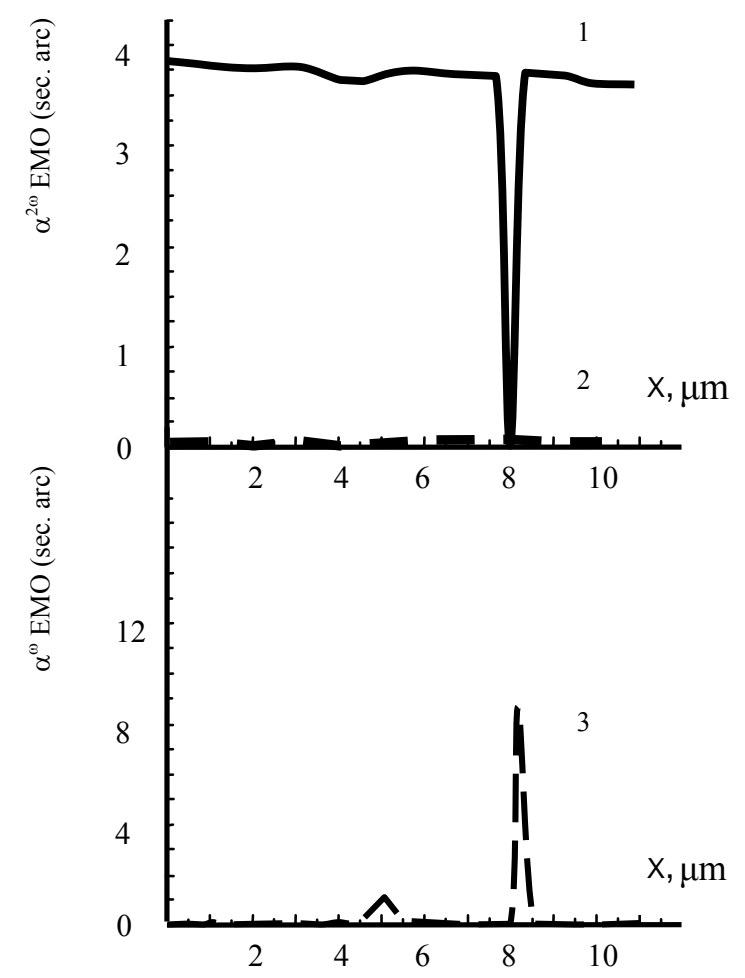

Figure 3. Dependences of the nonlinear $\left(\alpha^{2 \omega}-1\right)$ and linear $\left(\alpha^{\omega}-3\right)$ components of EMOE from positions of scanning diaphragm $(\mathrm{X})$ in the central areas of separate magnetic domain after thermal influence to the sample $(\mathrm{H}=\mathbf{0}$ Oe and $E=3,2 \mathrm{kV} / \mathrm{cm}$ ). Curve (2) was measured when $\mathrm{H}=140 \mathrm{Oe}$ and $\mathrm{E}=3,2 \mathrm{kV} / \mathrm{cm}$.

it is possible to carry out the control of the films on defects presence in them and to define their positions and that can matter for application. i.e., the local EMOE picture from the separate sites of the domain must be more informative than an averaged one in the multidomain case. Thermal influence on electromagneto-optical dependences of the film has not led to essential changes in character of EMOE.

\section{References}

[1] J. F. Dillon, "Optical Properties of Several Ferrimagnetic Garnets," Journal of Applied Physics, Vol. 29, No. 3, 1958, pp. 539-543. doi:10.1063/1.1723215

[2] L. E. Helseth, R. W. Hansen, E. I. Il'yashenko, M. Baziljevich and T. H. Johansen, "Faraday Rotation Spectra of Bismuth-Substituted Ferrite Garnet Films with in-Plane Magnetization," Physical Review B, Vol. 64, No. , 2001, pp. 174406-174411.

[3] G. B. Scott, D. E. Lacklison and J. L. Page, "Absorption Spectra of $\mathrm{Y}_{3} \mathrm{Fe}_{5} \mathrm{O}_{12}$ (YIG) and $\mathrm{Y}_{3} \mathrm{Ga}_{5} \mathrm{O}_{12}: \mathrm{Fe}^{3+}$," Physical Review B, Vol. 10, 1974, pp. 971-976. doi:10.1103/PhysRevB.10.971 
[4] S. H. Wemple, S. L. Blank, J. A. Seman and W. A. Biolsi, "Optical Properties of Epitaxial Iron Garnet Thin Films," Physical Review B, Vol. 9, No. 5, 1974, pp. 2134-2138. doi:10.1103/PhysRevB.9.2134

[5] S. Wittekoek, T. J. A. Popma, J. M. Robertson and P. F. Bongers, "Magneto-Optic Spectra and the Dielectric Tensor Elements of Bismuth-Substituted Iron Garnets at Photon Energies between 2.2-5.2 eV," Physical Review B, Vol. 12, No. 7, 1975, pp. 2777-2780. doi:10.1103/PhysRevB.12.2777

[6] P. Hansen, C. P. Klages, J. Schuldt and K. Witter, "Magnetic and Magneto-Optical Properties of BismuthSubstituted Lutetium Iron Garnet Films," Physical Review B, Vol. 31, No. 9, 1985, pp. 5858-5862. doi:10.1103/PhysRevB.31.5858

[7] T. H. O'Dell, "The Electrodynamics of Magnetoelectric Media," Philosophical Magazine, Vol. 7, No. 82, 1967, pp. 1653-1669. doi:10.1080/14786436208213701

[8] V. E. Koronovskyy, S. M. Ryabchenko and V. F. Kovalenko, "Electromagneto-Optical Effects on Local Areas of Ferrite-Garnet Film," Physical Review B, Vol. 71, 2005, pp. 172402-172405. doi:10.1103/PhysRevB.71.172402
[9] V. E. Koronovskyy and A. A. Kalenichenko, "Electromagneto-Optical Effect in Ferrimagnetic/Piezoelectric Structure," Applied Physics A, Vol. 97, 2009, pp. 933-936. doi:10.1007/s00339-009-5365-1

[10] V. E. Koronovskyy, "Influence of Powerful Laser Irradiation on Electromagneto-Optical Dependences of Yttrium Iron Garnets," Journal of Applied Physics, Vol. 106, No. 6, 2009, pp. 063914-1-063914-3. doi:10.1063/1.3224962

[11] B. B. Krichevtsov, R. V. Pisarev and A. G. Selitskij, "The Electromagneto-Optical Effect in Yttrium Iron Garnets $\mathrm{Y}_{3} \mathrm{Fe}_{5} \mathrm{O}_{12}$," JETP Letters, Vol. 41, 1985, pp. 317- 319.

[12] G. Aubert, "A Novel Approach of the Magnetoelectric Effects in Ferrimagnets," Journal of Magnetism and Magnetic Materials, Vol. 31-34, 1983, pp. 767-768.

[13] G. Velleaud, B. Sangare, M. Mercier and G.Aubert, "Magnetoelectric Properties of Yttrium Iron Garnet," Solid State Communications, Vol. 52, 1984, pp. 71-74.

[14] V. E.Koronovskyy and B. Y. Scherbak, "Thermal Influence on Magnetoelectric Characteristics of Yttrium Iron Garnet Epitaxial Films," Functional Materials, Vol. 16, No. 1, 2010, pp. 59-61. 